\title{
Rationality, irrationality, and Wilf equivalence in generalized factor order
}

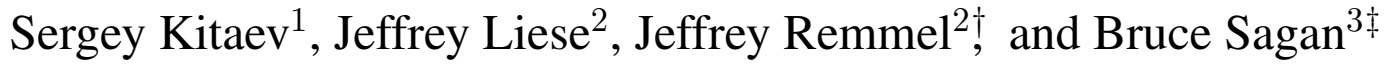 \\ ${ }^{1}$ Institute of Mathematics, Reykjavík University, IS-103 Reykjavik, Iceland \\ ${ }^{2}$ Department of Mathematics, University of California, San Diego, La Jolla, CA 92093-0112, USA \\ ${ }^{3}$ Department of Mathematics, Michigan State University, East Lansing, MI 48824-1027, USA
}

\begin{abstract}
Let $P$ be a partially ordered set and consider the free monoid $P^{*}$ of all words over $P$. If $w, w^{\prime} \in P^{*}$ then $w^{\prime}$ is a factor of $w$ if there are words $u, v$ with $w=u w^{\prime} v$. Define generalized factor order on $P^{*}$ by letting $u \leq w$ if there is a factor $w^{\prime}$ of $w$ having the same length as $u$ such that $u \leq w^{\prime}$, where the comparison of $u$ and $w^{\prime}$ is done componentwise using the partial order in $P$. One obtains ordinary factor order by insisting that $u=w^{\prime}$ or, equivalently, by taking $P$ to be an antichain.
\end{abstract}

Given $u \in P^{*}$, we prove that the language $\mathcal{F}(u)=\{w: w \geq u\}$ is accepted by a finite state automaton. If $P$ is finite then it follows that the generating function $F(u)=\sum_{w \geq u} w$ is rational. This is an analogue of a theorem of Björner and Sagan for generalized subword order.

We also consider $P=\mathbb{P}$, the positive integers with the usual total order, so that $\mathbb{P}^{*}$ is the set of compositions. In this case one obtains a weight generating function $F(u ; t, x)$ by substituting $t x^{n}$ each time $n \in \mathbb{P}$ appears in $F(u)$. We show that this generating function is also rational by using the transfer-matrix method. Words $u, v$ are said to be Wilf equivalent if $F(u ; t, x)=F(v ; t, x)$ and we can prove various Wilf equivalences combinatorially.

Björner found a recursive formula for the Möbius function of ordinary factor order on $P^{*}$. It follows that one always has $\mu(u, w)=0, \pm 1$. Using the Pumping Lemma we show that the generating function $M(u)=\sum_{w \geq u}|\mu(u, w)| w$ can be irrational.

Résumé. Soit $P$ un ensemble partiellement ordoné. Nous considérons le monoïde libre $P^{*}$ de tous les mots utilisant $P$ comme alphabet. Si $w, w^{\prime} \in P^{*}$, on dit que $w^{\prime}$ est un facteur de $w$ s'il y a des mots $u, v$ avec $w=u w^{\prime} v$. Nous definissons l'ordre facteur généralisé sur $P^{*}$ par: $u \leq w$ s'il y a un facteur $w^{\prime}$ de $w$ ayant la même longueur que $u$ tel que $u \leq w^{\prime}$, où la comparison de $u$ avec $w^{\prime}$ est faite lettre par lettre utilisant l'ordre en $P$. On obtient l'ordre facteur usuel si on insiste que $u=w^{\prime}$ ou, ce qui est la même chose, en prenant $P$ comme antichaîne.

Pour n'importe quel $u \in P^{*}$, nous démontrons que le langage $\mathcal{F}(u)=\{w: w \geq u\}$ est accepté par un automaton avec un nombre fini d'états. Si $P$ est fini, ca implique que la fonction génératrice $F(u)=\sum_{w \geq u} w$ est rationnelle. Björner et Sagan ont démontré le théorème analogue pour l'ordre où, en la définition au-dessus, $w^{\prime}$ est un sous-mot de $w$.

Nous considérons aussi le cas $P=\mathbb{P}$, les entiers positifs avec l'ordre usuel, donc $P^{*}$ est l'ensemble des compositions. En ce cas on obtient une fonction génératrice pondéré $F(u ; t, x)$ en remplaçant $t x^{n}$ chaque fois on trouve $n \in \mathbb{P}$

\footnotetext{
$\dagger$ Partially supported by NSF grant DMS 0654060

${ }^{\ddagger}$ Work partially done while a Program Officer at NSF, The views expressed are not necessarily those of the NSF.
} 
en $F(u)$. Nous démontrons que cette fonction génératrice est aussi rationnelle en utilisant la Méthode Matrice de Tranfert. On dit que let mots $u, v$ sont Wilf-équivalents si $F(u ; t, x)=F(v ; t, x)$. Nous pouvons démontré quelques équivalances dans une manière combinatorie.

Björner a trouvé une formule recursive pour la fonction Möbius de l'ordre facteur usuel sur $P^{*}$. Cette formule implique qu'on a toujours $\mu(u, w)=0, \pm 1$. En utilisant le Lemme de Pompage, nous démontrons que la fonction génératrice $M(u)=\sum_{w \geq u}|\mu(u, w)| w$ peut être irrationnelle.

Keywords: composition, factor order, finite state automaton, partially ordered set, rational generating function, Wilf equivalence

\section{Introduction and definitions}

Let $P$ be a set and consider the corresponding free monoid or Kleene closure of all words over $P$ :

$$
P^{*}=\left\{w=w_{1} w_{2} \ldots w_{\ell}: \ell \geq 0 \text { and } w_{i} \in P \text { for all } i\right\}
$$

Let $\epsilon$ be the empty word and for any $w \in P^{*}$ we denote its cardinality or length by $|w|$. Given $w, w^{\prime} \in$ $P^{*}$, we say that $w^{\prime}$ is a factor of $w$ if there are words $u, v$ with $w=u w^{\prime} v$, where adjacency denotes concatenation. For example, $w^{\prime}=322$ is a factor of $w=12213221$ starting with the fifth element of $w$. Factor order on $P^{*}$ is the partial order obtained by letting $u \leq_{\text {fo }} w$ if and only if there is a factor $w^{\prime}$ of $w$ with $u=w^{\prime}$.

Now suppose that we have a poset $(P, \leq)$. We define generalized factor order on $P^{*}$ by letting $u \leq$ gfo $w$ if there is a factor $w^{\prime}$ of $w$ such that

(a) $|u|=\left|w^{\prime}\right|$, and

(b) $u_{i} \leq w_{i}^{\prime}$ for $1 \leq i \leq|u|$.

We call $w^{\prime}$ an embedding of $u$ into $w$, and if the first element of $w^{\prime}$ is the $j$ th element of $w$, we call $j$ an embedding index of $u$ into $w$. We also say that in this embedding $u_{i}$ is in position $j+i-1$. To illustrate, suppose $P=\mathbb{P}$, the positive integers with the usual order relation. If $u=322$ and $w=12213431$ then $u \leq_{\text {gfo }} w$ because of the embedding factor $w^{\prime}=343$ which has embedding index 5, and the two 2's of $u$ are in positions 6 and 7. Note that we obtain ordinary factor order by taking $P$ to be an antichain. Also, we will henceforth drop the subscript gfo since context will make it clear what order relation is meant. Generalized factor order is the focus of this extended abstract.

Returning to the case where $P$ is an arbitrary set, let $\mathbb{Z}\langle\langle P\rangle\rangle$ be the algebra of formal power series with integer coefficients and having the elements of $P$ as noncommuting variables. In other words,

$$
\mathbb{Z}\langle\langle P\rangle\rangle=\left\{f=\sum_{w \in P^{*}} c_{w} w: c_{w} \in \mathbb{Z} \text { for all } w\right\} .
$$

If $f \in \mathbb{Z}\langle\langle P\rangle\rangle$ has no constant term, i.e., $c_{\epsilon}=0$, then define

$$
f^{*}=\epsilon+f+f^{2}+f^{3}+\cdots=(\epsilon-f)^{-1} .
$$


(We need the restriction on $f$ to make sure that the sums are well defined as formal power series.) We say that $f$ is rational if it can be constructed from the elements of $P$ using only a finite number of applications of the algebra operations and the star operation.

A language is any $\mathcal{L} \subseteq P^{*}$. It has an associated generating function

$$
f_{\mathcal{L}}=\sum_{w \in \mathcal{L}} w
$$

The language $\mathcal{L}$ is regular if $f_{\mathcal{L}}$ is rational.

Consider generalized factor order on $P^{*}$ and fix a word $u \in P^{*}$. There is a corresponding language and generating function

$$
\mathcal{F}(u)=\{w: w \geq u\} \quad \text { and } \quad F(u)=\sum_{w \geq u} w
$$

Our first result is as follows.

Theorem 1.1 If $P$ is a finite poset and $u \in P^{*}$ then $F(u)$ is rational.

Theorem 1.1 is an analogue of a result of Björner and Sagan [4] for generalized subword order on $P^{*}$. Generalized subword order is defined exactly like generalized factor order except that $w^{\prime}$ is only required to be a subword of $w$, i.e., the elements of $w^{\prime}$ need not be consecutive in $w$. For related results, also see Goyt [5].

Given any set, $P$, a nondeterministic finite automaton or NFA over $P$ is a digraph (directed graph) $\Delta$ with vertices $V$ and arcs $\vec{E}$ having the following properties.

1. The elements of $V$ are called states and $|V|$ is finite.

2. There is a designated initial state $\alpha$ and a set $\Omega$ of final states.

3. Each $\operatorname{arc}$ of $\vec{E}$ is labeled with an element of $P$.

Given a (directed) path in $\Delta$ starting at $\alpha$, we construct a word in $P^{*}$ by concatenating the elements on the arcs on the path in the order in which they are encountered. The language accepted by $\Delta$ is the set of all such words which are associated with paths ending in a final state. It is a well-known theorem that, for $|P|$ finite, a language $\mathcal{L} \subseteq P^{*}$ is regular if and only if there is a NFA accepting $\mathcal{L}$. (See, for example, the text of Hopcroft and Ullman [6, Chapter 2].)

We will demonstrate Theorem 1.1 by constructing a NFA accepting the language for $F(u)$. This will be done in the next section. In fact, the NFA still exists even if $P$ is infinite, suggesting that more can be said about the generating function in this case.

We are particularly interested in the case of $P=\mathbb{P}$ with the usual order relation. So $\mathbb{P}^{*}$ is just the set of compositions (ordered integer partitions). Given $w=w_{1} w_{2} \ldots w_{\ell} \in \mathbb{P}^{*}$, we define its norm to be

$$
\Sigma(w)=w_{1}+w_{2}+\cdots+w_{\ell} .
$$

Let $t, x$ be commuting variables. Replacing each $n \in w$ by $t x^{n}$, we get an associated monomial called the weight of $w$

$$
w t(w)=t^{|w|} x^{\Sigma(w)} .
$$


For example, if $w=213221$ then

$$
w t(w)=t x^{2} \cdot t x \cdot t x^{3} \cdot t x^{2} \cdot t x^{2} \cdot t x=t^{6} x^{11} .
$$

We also have the associated weight generating function

$$
F(u ; t, x)=\sum_{w \geq u} w t(w)
$$

Our NFA will demonstrate, via the transfer-matrix method, that this is also a rational function of $t$ and $x$. The details will be given in Section 3 .

Call $u, w \in \mathbb{P}^{*}$ Wilf equivalent if $F(u ; t, x)=F(v ; t, x)$. This definition is modelled on the one used in the theory of pattern avoidance. See the survey article of Wilf [8] for more information about this subject. Section 4 is devoted to stating various Wilf equivalences all of which can be proved combinatorially.

Björner [2] gave a recursive formula for the Möbius function of (ordinary) factor order. It follows from his theorem that $\mu(u, w)=0, \pm 1$ for all $u, w \in P^{*}$. Using the Pumping Lemma [6. Lemma 3.1] we show that there are finite sets $P$ and $u \in P^{*}$ such that the language

$$
\mathcal{M}(u)=\{w: \mu(u, w) \neq 0\}
$$

is not regular. This is done in Section 5 The final section is devoted to comments and open questions.

\section{Construction of automata}

We will now introduce another language which is related to $\mathcal{F}(u)$ and which will be useful in proving Theorem 1.1. We say that $u$ is a suffix (respectively, prefix) of $w$ if $w=v u$ (respectively, $w=u v$ ) for some word $v$. Let $\mathcal{S}(u)$ be all the $w \in \mathcal{F}(u)$ such that, in the definition of generalized factor order, the only possible choice for $w^{\prime}$ is a suffix of $w$. Let $S(u)$ be the corresponding generating function.

The next result follows easily from the definitions and so we omit the proof. In it, we will use the notation $Q$ to stand both for a subset of $P$ and for the generating function $Q=\sum_{a \in Q} a$. Context will make it clear which is meant.

Lemma 2.1 Let $P$ be any poset and let $u \in P^{*}$. Then we have the following relationships

$$
\mathcal{F}(u)=\mathcal{S}(u) P^{*} \quad \text { and } \quad F(u)=S(u)(\epsilon-P)^{-1}
$$

between the languages and between the generating functions.

We will now prove that the two languages we have defined are accepted by NFAs. An example follows the proof so the reader may want to read it in parallel.

Theorem 2.2 Let $P$ be any poset and let $u \in P^{*}$. Then there are NFAs accepting $\mathcal{F}(u)$ and $\mathcal{S}(u)$.

Proof: We first construct an NFA, $\Delta$, for $\mathcal{S}(u)$. Let $\ell=|u|$. The states of $\Delta$ will be all subsets $T$ of $\{1, \ldots, \ell\}$. The initial state is $\emptyset$. Let $w=w_{1} \ldots w_{m}$ be the word corresponding to a path from $\emptyset$ to $T$. The NFA will be constructed so that if the path is continued, the only possible embedding indices are those in the set $\{m-t+1: t \in T\}$. In other words, for each $t \in T$ we have

$$
u_{1} u_{2} \ldots u_{t} \leq w_{m-t+1} w_{m-t+2} \ldots w_{m}
$$




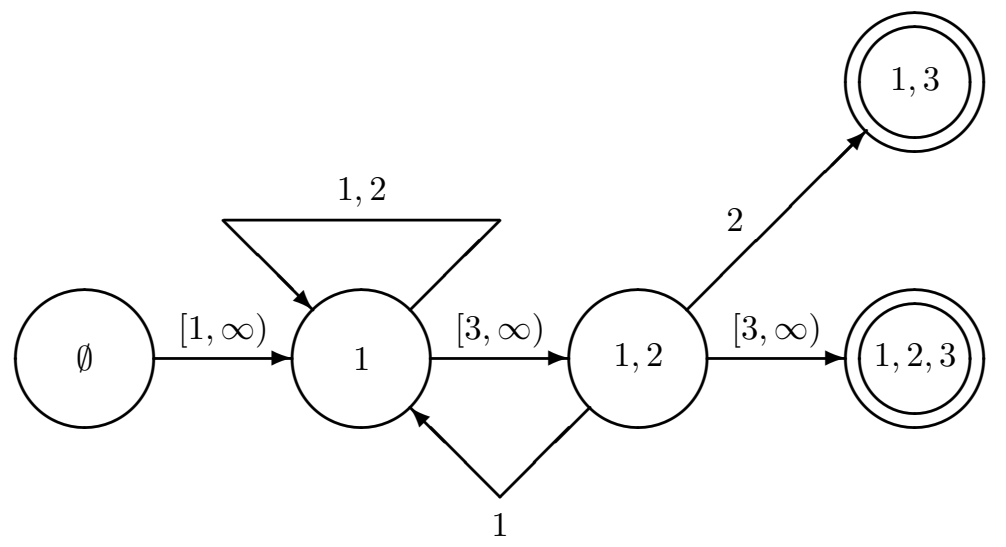

Fig. 1: A NFA accepting $\mathcal{S}(132)$

for each $t \in\{1, \ldots, \ell\}-T$ this inequality does not hold, and $u \not \leq w^{\prime}$ for any factor $w^{\prime}$ of $w$ starting at an index smaller then $m-\ell+1$. From this description it is clear that the final states should be those containing $\ell$.

The definition of the arcs of $\Delta$ is forced by the interpretation of the states. There will be no arcs out of a final state. If $T$ is a nonfinal state and $a \in P$ then there will be an $\operatorname{arc}$ from $T$ to

$$
T^{\prime}=\left\{t+1: t \in T \cup\{0\} \text { and } u_{t+1} \leq a\right\} .
$$

It is easy to see that 11 continues to hold for all $t^{\prime} \in T^{\prime}$ once we append $a$ to $w$. This finishes the construction of the NFA for $\mathcal{S}(u)$. To obtain an automaton for $\mathcal{F}(u)$, just add loops to the final states of $\Delta$, one for each $a \in P$.

As an example, consider $P=\mathbb{P}$ and $u=132$. We will do several things to simplify writing down the automaton. First of all, certain states may not be reachable by a path starting at the initial state. So we will not display such states. For example, we can not reach the state $\{2,3\}$ since $u_{1}=1 \leq w_{i}$ for any $i$ and so 1 will be in any state reachable from $\phi$. Also, given states $T$ and $U$ there may be many arcs from $T$ to $U$, each having a different label. So we will replace them by one arc bearing the set of labels of all such arcs. Finally, set braces will be dropped for readability. The resulting digraph is displayed in Figure 1.

Consider what happens as we build a word $w$ starting from the initial state $\emptyset$. Since $u_{1}=1$, any element of $\mathbb{P}$ could be the first element of an embedding of $u$ into $w$. That is why every element of the interval $[1, \infty)=\mathbb{P}$ produces an arrow from the initial state to the state $\{1\}$. Now if $w_{2} \leq 2$, then an embedding of $u$ could no longer start at $w_{1}$ and so these elements give loops at the state $\{1\}$. But if $w_{2} \geq 3$ then an embedding could start at either $w_{1}$ or at $w_{2}$ and so the corresponding arcs all go to the state $\{1,2\}$. The rest of the automaton is explained similarly.

As an immediate consequence of the previous theorem we get the following result which includes Theorem 1.1 
Theorem 2.3 Let $P$ be a finite poset and let $u \in P^{*}$. Then the generating functions $F(u)$ and $S(u)$ are rational.

\section{The positive integers}

If $P=\mathbb{P}$ then Theorem 2.3 no longer applies to the generating functions $F(u)$ and $S(u)$. However, we can still show rationality of the weight generating function $F(u ; t, x)$ as defined in the introduction. Similarly, we will see that the series $S(u ; t, x)=\sum_{w \in \mathcal{S}(u)} w t(w)$ is rational.

Note first that Lemma 2.1 still holds for $\mathbb{P}$ and can be made more explicit in this case. Extend the function $w t$ to all of $\mathbb{Z}\langle\langle\mathbb{P}\rangle\rangle$ by letting it act linearly. Then

$$
w t(\epsilon-\mathbb{P})^{-1}=\frac{1}{1-\sum_{n \geq 1} t x^{n}}=\frac{1}{1-t x /(1-x)}=\frac{1-x}{1-x-t x} .
$$

We now plug this into the lemma just cited.

Corollary 3.1 We have $F(u ; t, x)=(1-x) S(u ; t, x) /(1-x-t x)$.

It follows that if one of these three series is rational then the other one is as well.

We will now use the NFA, $\Delta$, constructed in Theorem 2.2 to show that $S(u ; t, x)$ is rational. This is essentially an application of the transfer-matrix method. See the text of Stanley [7, Section 4.7] for more information about this technique. The transfer matrix $M$ for $\Delta$ has rows and columns indexed by the states with

$$
M_{T, U}=\sum_{n} w t(n)
$$

where the sum is over all $n$ which appear as labels on the $\operatorname{arcs}$ from $T$ to $U$. For example, consider the case where $w=132$ as done at the end of the previous section. If we list the states in the order

$$
\emptyset,\{1\},\{1,2\},\{1,3\},\{1,2,3\}
$$

then the transfer matrix is

$$
M=\left[\begin{array}{ccccc}
0 & \frac{t x}{1-x} & 0 & 0 & 0 \\
0 & t\left(x+x^{2}\right) & \frac{t x^{3}}{1-x} & 0 & 0 \\
0 & t x & 0 & t x^{2} & \frac{t x^{3}}{1-x} \\
0 & 0 & 0 & 0 & 0 \\
0 & 0 & 0 & 0 & 0
\end{array}\right]
$$

Now $M^{k}$ has entries $M_{T, U}^{k}=\sum_{w} w t(w)$ where the sum is over all words $w$ corresponding to a directed walk of length $k$ from $T$ to $U$. So to get the weight generating function for walks of all lengths one considers $\sum_{k \geq 0} M^{k}$. Note that this sum converges in the algebra of matrices over the formal power series algebra $\mathbb{Z}[[t, x]]$ because none of the entries of $M$ has a constant term. It follows that

$$
L:=\sum_{k \geq 0} M^{k}=(I-M)^{-1}=\frac{\operatorname{adj}(I-M)}{\operatorname{det}(I-M)}
$$


where adj denotes the adjoint.

Now

$$
S(u ; t, x)=\sum_{T} L_{\emptyset, T}
$$

where the sum is over all final states of $\Delta$. So it suffices to show that each entry of $L$ is rational. From equation (2), this reduces to showing that each entry of $M$ is rational. So consider two given states $T, U$. If $T$ is final then we are done since the $T$ th row of $M$ is all zeros. If $T$ is not final, then consider

$$
T^{\prime}=\{t+1: t \in T \cup\{0\}\} .
$$

If $U=T^{\prime}$ then there will be an $N \in \mathbb{P}$ such that all the arcs out of $T$ with labels $n \geq N$ go to $T^{\prime}$. So $M_{T, T^{\prime}}$ will contain $\sum_{n>N} t x^{n}=t x^{N} /(1-x)$ plus a finite number of other terms of the form $t x^{m}$. Thus this entry is rational. If $U \neq T^{\prime}$, then there will only be a finite number of $\operatorname{arcs}$ from $T$ to $U$ and so $M_{T, U}$ will actually be a polynomial. This shows that every entry of $M$ is rational and we have proved, with the aid of the remark following Corollary 3.1 , the following result.

Theorem 3.2 If $u \in \mathbb{P}^{*}$ then $F(u ; t, x)$ and $S(u ; t, x)$ are rational.

\section{Wilf equivalence}

Recall that $u, v \in \mathbb{P}^{*}$ are Wilf equivalent, written $u \sim v$, if $F(u ; t, x)=F(v ; t, x)$. By Corollary 3.1. this is equivalent to $S(u ; t, x)=S(v ; t, x)$. It follows that to prove Wilf equivalence, it suffices to find a weight-preserving bijection $f: \mathcal{L}(u) \rightarrow \mathcal{L}(v)$ where $\mathcal{L}=\mathcal{F}$, or $\mathcal{S}$. Since $\sim$ is an equivalence relation, we can talk about the Wilf equivalence class of $u$ which is $\{w: w \sim u\}$. It is worth noting that the automata for the words in a Wilf equivalence class need not bear a resemblance to each other. Part of the motivation for this section is to try to explain as many Wilf equivalences as possible between permutations.

First of all, we consider three operations on words in $\mathbb{P}^{*}$. The reversal of $u=u_{1} \ldots u_{\ell}$ is $u^{r}=$ $u_{\ell} \ldots u_{1}$. It will also be of interest to consider $1 u$, the word gotten by prepending one to $u$. Finally, we will look at $u^{+}$which is gotten by increasing each element of $u$ by one. It is not hard to give combinatorial proofs for the three facts in the next theorem, but due to space limitations we will only do so for the second.

Theorem 4.1 We have the following Wilf equivalences.

(a) $u \sim u^{r}$,

(b) if $u \sim v$ then $1 u \sim 1 v$,

(c) if $u \sim v$ then $u^{+} \sim v^{+}$.

Proof: (b) We can assume we are given a weight-preserving bijection $f: \mathcal{S}(u) \rightarrow \mathcal{S}(v)$. Since 1 is the minimal element of $\mathbb{P}$,

$$
\mathcal{S}(1 u)=\left\{w \in \mathbb{P}^{*}: w_{2} w_{3} \ldots w_{|w|} \in S(u)\right\} .
$$

So $f$ induces a weight-preserving bijection $g: \mathcal{S}(1 u) \rightarrow \mathcal{S}(1 v)$ defined by

$$
g\left(w_{1} w_{2} \ldots w_{n}\right)=w_{1} f\left(w_{2} \ldots w_{n}\right)
$$


and we are done.

Applying the previous result, we can obtain all the Wilf equivalences in the symmetric groups $\mathfrak{S}_{2}$ and $\mathfrak{S}_{3}$. In $\mathfrak{S}_{2}$ we have $12 \sim 21$ by (a). So $23 \sim 32$ by (c) and $123 \sim 132$ by (b). Continuing in this way, we obtain

$$
123 \sim 321 \sim 132 \sim 231 \text { and } 213 \sim 312 .
$$

These two groups are indeed in different equivalence classes as one can use equation (2) to compute that

$$
S(123 ; t, x)=\frac{t^{3} x^{6}}{(1-x)^{2}\left(1-x-t x+t x^{3}-t^{2} x^{4}\right)}
$$

while

$$
S(213 ; t, x)=\frac{t^{3} x^{6}\left(1+t x^{3}\right)}{(1-x)\left(1-x+t^{2} x^{4}\right)\left(1-x-t x+t x^{3}-t^{2} x^{4}\right)} .
$$

We will need a new result to explain some of the equivalences in $\mathfrak{S}_{4}$ such as $2134 \sim 2143$. This is done by the next result which, in conjunction with Theorem 4.1, can be used to derive all of the equivalences in $\mathfrak{S}_{4}$. We omit the proof due to space limitations.

Theorem 4.2 Let $x, y, z \in\{1, \ldots, m\}^{*}$ and suppose $n>m$. Then

$$
\text { xmynz } \sim \text { xnymz. }
$$

\section{The Möbius function}

We will now show that the language for the Möbius function of ordinary factor order is not regular. This is somewhat surprising because Björner and Reutenauer [3] showed that this language is regular if one considers ordinary subword order, and then Björner and Sagan [4] extended this result to generalized subword order. We will begin by reviewing some basic facts about Möbius functions. The reader wishing more details can consult [7, Chapter 3].

For any poset $P$, the incidence algebra of $P$ over the integers is

$$
I(P)=\{\alpha: P \times P \rightarrow \mathbb{Z}: \alpha(a, b)=0 \text { if } a \not \leq b\} .
$$

This set is an algebra whose multiplication is given by convolution $(\alpha * \beta)(a, b)=\sum_{c \in P} \alpha(a, c) \beta(c, b)$. It is easy to see that the identity for this operation is the Kronecker delta

$$
\delta(a, b)= \begin{cases}1 & \text { if } a=b, \\ 0 & \text { else. }\end{cases}
$$

So it is possible for incidence algebra elements to have multiplicative inverses.

One of the simplest elements of $I(P)$ is the zeta function

$$
\zeta(a, b)= \begin{cases}1 & \text { if } a \geq b \\ 0 & \text { else }\end{cases}
$$

Note that $F(u)$ can be rewritten as $F(u)=\sum_{w \in P^{*}} \zeta(u, w) w$. It turns out that $\zeta$ has a convolutional inverse $\mu$ in $I(P)$. This function is important in enumerative and algebraic combinatorics. Björner [2] 
has given a formula for $\mu$ in ordinary factor order which we will need. To describe this result, we must make some definitions. The dominant outer factor of $w$, denoted $o(w)$, is the longest word other than $w$ which is both a prefix and a suffix of $w$. Note that we may have $o(w)=\epsilon$. The dominant inner factor of $w=w_{1} \ldots w_{\ell}$, written $i(w)$, is $w_{2} \ldots w_{\ell-1}$. Finally, a word is flat if all its elements are equal. For example, $w=a b b a a b b$ has $o(w)=a b b$ and $i(w)=b b a a b$.

Theorem 5.1 (Björner) In (ordinary) factor order, if $u \leq w$ then

$$
\mu(u, w)= \begin{cases}\mu(u, o(w)) & \text { if }|w|-|u|>2 \text { and } u \leq o(w) \leq i(w), \\ 1 & \text { if }|w|-|u|=2 \text {, w is not flat, and } u=o(w) \text { or } i(w), \\ (-1)^{|w|-|u|} & \text { if }|w|-|u|<2, \\ 0 & \text { otherwise. }\end{cases}
$$

Continuing the example

$$
\mu(b, a b b a a b b)=\mu(b, a b b)=1 .
$$

Note that this description is inductive. It also implies that $\mu(u, w)$ is \pm 1 or 0 for all $u, w$ in factor order.

We will show that the language $\mathcal{M}(u)=\{w: \mu(u, w) \neq 0\}$ need not be regular. To do this, we will need the Pumping Lemma which we now state. A proof can be found in [6, pp. 55-56].

Lemma 5.2 (Pumping Lemma) Let $\mathcal{L}$ be a regular language. Then there is a constant $n \geq 1$ such that any $z \in \mathcal{L}$ can be written as $z=$ uvw satisfying

$$
\begin{aligned}
& \text { 1. }|u v| \leq n \text { and }|v| \geq 1 \text {, } \\
& \text { 2. } u v^{i} w \in \mathcal{L} \text { for all } i \geq 0 .
\end{aligned}
$$

Roughly speaking, any word in a regular language has a prefix of bounded length such that pumping up the end of the prefix keeps one in the language.

Theorem 5.3 Consider (ordinary) factor order where $P=\{a, b\}$. Then $\mathcal{M}(a)$ is not regular.

Proof: Suppose, to the contrary, that $\mathcal{M}(a)$ is regular and let $n$ be the constant guaranteed by the pumping lemma. We will derive a contradiction by letting $z=a b^{n} a b^{n} a$ where, as usual, $b^{n}$ represents the letter $b$ repeated $n$ times.

First we show that $z \in \mathcal{M}(a)$. Indeed, $o(z)=a b^{n} a$ and $i(z)=b^{n} a b^{n}$ which implies that $a \leq o(z) \not \leq$ $i(z)$. So we are in the first case of Björner's formula and $\mu(a, z)=\mu\left(a, a b^{n} a\right)$. Repeating this analysis with $a b^{n} a$ in place of $z$ gives $\mu(a, z)=\mu(a, a)=1$. Hence $z \in \mathcal{M}(a)$ as promised.

Now pick any prefix $u v$ of $z$ as in the Pumping Lemma. There are two cases. The first is if $u \neq \epsilon$. So $v=b^{j}$ for some $j$ with $1 \leq j<n$. Picking $i=2$, we conclude that $z^{\prime}=u v^{2} w=a b^{n+j} a b^{n} a$ is in $\mathcal{M}(a)$. But $o\left(z^{\prime}\right)=a$ and $i\left(z^{\prime}\right)=b^{n+j} a b^{n}$. Thus $\left|z^{\prime}\right|-|a|>2$ and $a \leq o\left(z^{\prime}\right) \leq i\left(z^{\prime}\right)$, so $z^{\prime}$ does not fall into any of the first three cases of Björner's formula. This implies that $\mu\left(a, z^{\prime}\right)=0$ and hence $z^{\prime} \notin \mathcal{M}(a)$, which is a contradiction in this case.

The second possibility is that $u=\epsilon$ and $v=a b^{j}$ for some $0 \leq j<n$. Similar considerations to those in the previous paragraph show that if we take $z^{\prime}=u v^{2} w$ then $\mu\left(a, z^{\prime}\right)=0$ again. So we have a contradiction as before and the theorem is proved. 


\section{Comments, conjectures, and open questions}

\subsection{Mixing factors and subwords}

It is possible to create languages using combinations of factors and subwords. This is an idea that was first studied by Babson and Steingrímsson [1] in the context of pattern avoidance in permutations. Many of the results we have proved can be generalized in this way. We will indicate how this can be done for Theorem 2.2

A pattern $p$ over $\mathrm{P}$ is a word in $P^{*}$ where certain pairs of adjacent elements have been overlined (barred). For example, in the pattern $p=1 \overline{133} 24 \overline{61}$ the pairs 13,33 , and 61 have been overlined. If $w \in P^{*}$ we will write $\bar{w}$ for the pattern where every pair of adjacent elements in $w$ is overlined. So every pattern has a unique factorization of the form $p=\overline{y_{1}} \overline{y_{2}} \ldots \overline{y_{k}}$. In the preceding example, the factors are $y_{1}=1, y_{2}=133, y_{3}=2, y_{4}=4$, and $y_{5}=61$.

If $p=\overline{y_{1}} \overline{y_{2}} \ldots \overline{y_{k}}$ is a pattern and $w \in P^{*}$ then $p$ embeds into $w$, written $p \rightarrow w$, if there is a subword $w^{\prime}=z_{1} z_{2} \ldots z_{k}$ of $w$ where, for all $i$

1. $z_{i}$ is a factor of $w$ with $\left|z_{i}\right|=\left|y_{i}\right|$, and

2. $y_{i} \leq z_{i}$ in generalized factor order.

For example $\overline{32} 4 \rightarrow 14235$ and there is only one embedding, namely 425 . For any pattern $p$, define the language

$$
\mathcal{F}(p)=\left\{w \in P^{*}: p \rightarrow w\right\}
$$

and similarly for $\mathcal{S}(p)$. The next result generalizes Theorem 2.2 to an arbitrary pattern. It is proved by pasting together automata like those constructed in that theorem.

Theorem 6.1 Let $P$ be any poset and let $p$ be a pattern over $P$. Then there are NFAs accepting $\mathcal{F}(p)$ and $\mathcal{S}(p)$.

\subsection{Rationality for infinite posets}

It would be nice to have a criterion that would imply rationality even for some infinite posets $P$. To this end, let $\mathbf{x}=\left\{x_{1}, \ldots, x_{m}\right\}$ be a set of commuting variables and consider the formal power series algebra $\mathbb{Z}[[\mathbf{x}]]$. Suppose we are given a function

$$
w t: P \rightarrow \mathbb{Z}[[\mathbf{x}]]
$$

which then defines a weighting of words $w=w_{1} \ldots w_{\ell} \in P^{*}$ by

$$
w t(w)=\prod_{i=1}^{m} w t\left(w_{i}\right)
$$

To make sure our summations will be defined in $\mathbb{Z}[[\mathbf{x}]]$, we assume that there are only finitely many $w$ of any given weight and call such a weight function regular.

For $u \in P^{*}$, let

$$
F(u ; \mathbf{x})=\sum_{w \geq u} w t(w)
$$


and similarly for $S(u ; \mathbf{x})$. Suppose we want to make sure that $S(u ; \mathbf{x})$ is rational. As done in $\operatorname{Section} 3$. we can consider a transfer matrix with entries

$$
M_{T, U}=\sum_{a} w t(a)
$$

where the sum is over all $a \in P$ occurring on arcs from $T$ to $U$. Equation (2) remains the same, so it suffices to make sure that $M_{T, U}$ is always rational.

If there is an arc labeled $a$ from $T$ to $U$ then we must have $U \subseteq T^{\prime}$ where $T^{\prime}$ is given in equation (3). Recalling the definition of $\Delta$ from the proof of Theorem 2.2, we see that the $a$ 's appearing in the previous sum are exactly those satisfying

1. $a \geq u_{t+1}$ for $t+1 \in U$, and

2. $a \nsupseteq u_{t+1}$ for $t+1 \in T^{\prime}-U$.

To state these criteria succinctly, for any subword $y$ of $u$ we write $a \succeq y$ (respectively, $a \nsucceq y$ ) if $a \geq b$ (respectively, $a \nsupseteq b$ ) for all $b \in y$. Finally, note that, from the proof of Theorem 2.2, similar transfer matrices can be constructed for $F(u ; \mathbf{x})$ and $A(u ; \mathbf{x})$. We have proved the following result which generalizes Theorem 3.2

Theorem 6.2 Let $P$ be a poset with a regular weight function $w t: P^{*} \rightarrow \mathbb{Z}[[\mathbf{x}]]$, and let $u \in P^{*}$. Suppose that for any two subwords $y$ and $z$ of $u$ we have

$$
\sum_{\substack{a \succeq y \\ a \succeq z}} w t(a)
$$

is a rational function. Then so are $F(u ; \mathbf{x})$ and $S(u ; \mathbf{x})$.

\subsection{Irrationality for infinite posets}

When $P$ is countably infinite it is possible for the generating functions we have considered to be irrational. As an example, fix a distinguished element $a \in P$. For each $A \subseteq P$ with $a \in A$, we define an order $\leq_{A}$ by insisting that the elements of $P-\{a\}$ form an antichain, and that $a \leq_{A} b$ if and only if $b \in A$. Consider the corresponding suffix language $\mathcal{S}_{A}(a)$. Clearly $\mathcal{S}_{A}(a)=(P-A)^{*} A$ and so no two of these languages are equal. It follows that the mapping $A \rightarrow \mathcal{S}_{A}(a)$ is injective. So one of the $\mathcal{S}_{A}(a)$ must be irrational since there are uncountably many possible $A$ but only countably many rational functions in $\mathbb{Z}\langle\langle P\rangle\rangle$.

\subsection{Wilf equivalence and strong equivalence}

There are a number of open problems and questions raised by our work on Wilf equivalence.

(1) If $u \sim v$, then must $v$ be a rearrangement of $u$ ? This is the case for all the Wilf equivalences we have proved.

(2) What about Wilf equivalence in $[m]^{*}$ where $[m]=\{1,2, \ldots, m\}$ ? Given a positive integer $m$, one can define Wilf equivalence of words $u, v \in[m]^{*}$ in the same way that we did for $\mathbb{P}^{*}$. We write $u \sim_{m} v$ for this relation. Is it true that $u \sim_{m} v$ if and only if $u \sim v$ ? 
(3) If $u^{+} \sim v^{+}$then is $u \sim v$ ? In other words, does the converse of Theorem 4.1 (c) hold? It is not hard to see that the converse of (b) is true.

(4) Find a theorem which, together with the results already proved, explains all the Wilf equivalences in $\mathfrak{S}_{5}$. We have a conjecture that would be helpful in this regard.

Conjecture 6.3 For any $a, b, c \in[2, \infty)$ we have

$$
a 1 b 2 c \sim a 2 b 1 c .
$$

(5) Is it always the case that the number of elements of $\mathfrak{S}_{n}$ Wilf equivalent to a given permutation is a power of 2? Our computations show that this is always true for $n \leq 5$.

\subsection{The language $\mathcal{M}(u)$}

We have shown that $\mathcal{M}(u)$ is not always regular and so the corresponding generating function $M(u)$ is not always rational. But this leaves open whether $\mathcal{M}(u)$ might fall into a more general class of languages such as context free grammars (CFGs). There is a Pumping Lemma for CFGs, see [6, Section 6.1]. So it is tempting to try and modify the proof of Theorem 5.3 to show that $\mathcal{M}(u)$ is not even a CFG. However, all our attempts in that direction have failed. Is $\mathcal{M}(u)$ a CFG or not?

\section{References}

[1] BABSON, E., AND SteingrímsSon, E. Generalized permutation patterns and a classification of the Mahonian statistics. Sém. Lothar. Combin. 44 (2000), Art. B44b, 18 pp. (electronic).

[2] BJÖRner, A. The Möbius function of factor order. Theoret. Comput. Sci. 117, 1-2 (1993), 91-98.

[3] Björner, A., And Reutenauer, C. Rationality of the Möbius function of subword order. Theoret. Comput. Sci. 98, 1 (1992), 53-63. Second Workshop on Algebraic and Computer-theoretic Aspects of Formal Power Series (Paris, 1990).

[4] Björner, A., And SAgan, B. E. Rationality of the Möbius function of a composition poset. Theoret. Comput. Sci. 359, 1-3 (2006), 282-298.

[5] Goyt, A. The Möbius function of a restricted composition poset. Preprint arXiv:0806.1500.

[6] Hopcroft, J. E., AND Ullman, J. D. Introduction to automata theory, languages, and computation. AddisonWesley Publishing Co., Reading, Mass., 1979. Addison-Wesley Series in Computer Science.

[7] Stanley, R. P. Enumerative Combinatorics. Vol. 1, vol. 49 of Cambridge Studies in Advanced Mathematics. Cambridge University Press, Cambridge, 1997. With a foreword by Gian-Carlo Rota, Corrected reprint of the 1986 original.

[8] WILF, H. S. The patterns of permutations. Discrete Math. 257, 2-3 (2002), 575-583. 